\title{
Technè
}

La science au service de l'histoire de l'art et de la préservation des biens culturels

$43 \mid 2016$

Une Europe de la recherche en sciences du patrimoine

\section{Initiatives européennes d'IPANEMA et du synchrotron SOLEIL pour l'étude des matériaux anciens}

European initiatives from the SOLEIL synchrotron and the IPANEMA center in ancient material research

Loïc Bertrand, Sebastian Schoeder, Sophie David et Mathieu Thoury

\section{OpenEdition}

\section{Journals}

Édition électronique

URL : http://journals.openedition.org/techne/723

DOI : $10.4000 /$ techne.723

ISSN : 2534-5168

Éditeur

C2RMF

Édition imprimée

Date de publication : 1 août 2016

Pagination : 75-78

ISBN : 978-2-7118-6338-9

ISSN : $1254-7867$

\section{Référence électronique}

Loïc Bertrand, Sebastian Schoeder, Sophie David et Mathieu Thoury, «Initiatives européennes d'IPANEMA et du synchrotron SOLEIL pour l'étude des matériaux anciens », Technè [En ligne], 43 2016, mis en ligne le 19 décembre 2019, consulté le 28 juillet 2020. URL : http:// journals.openedition.org/techne/723 ; DOI : https://doi.org/10.4000/techne.723

\section{(ब) $(\Theta \Theta$}

La revue Technè. La science au service de l'histoire de l'art et de la préservation des biens culturels est mise à disposition selon les termes de la Licence Creative Commons Attribution - Pas d'Utilisation Commerciale - Pas de Modification 4.0 International. 
Loïc Bertrand

Sebastian Schoeder

Sophie David

Mathieu Thoury

\section{Initiatives européennes d'IPANEMA et du synchrotron SOLEIL pour l'étude des matériaux anciens}

European initiatives from the SOLEIL synchrotron and the IPANEMA center in ancient material research

Résumé. Les particularités du rayonnement synchrotron, notamment sa très grande brillance et son spectre blanc des rayons $X$ à l'infrarouge lointain, en font une source de plus en plus employée pour l'étude de la composition chimique, des propriétés et de la réactivité des matériaux patrimoniaux. La construction de l'Institut photonique d'analyse non destructive européen des matériaux anciens, attenant au synchrotron SOLEIL, par l'État français et la Région Île-de-France permet un soutien fortement accru des équipes françaises et internationales travaillant à l'étude des matériaux anciens. Les allers-retours entre développement de méthodes et applications encouragent à la fois l'apparition de nouveaux instruments et un usage optimisé de techniques de spectroscopie et d'imagerie de pointe. Le développement d'IPANEMA sur le territoire de Paris-Saclay sinscrit dans le cadre plus global de la mise en place de l'infrastructure européenne E-RIHS, dédiée à l'étude du patrimoine culturel et naturel.

Mots-clés. Matériaux anciens, synchrotron, méthodes photoniques, imagerie, micro-échantillon.
Abstract. The specific features of synchrotron radiation, namely its very high brightness and its white spectrum from X-rays to far infrared light, make it an increasingly used source in the study of the chemical composition, properties and reactivity of ancient materials. Constructed by the French government and the Île-de-France region, adjacent to the SOLEIL synchrotron, IPANEMA (Institut photonique d'analyse non-destructive européen des matériaux anciens/European Institute of Non-Destructive Photonic Analysis of Ancient Materials), provides extensive additional support to French and international teams involved in research into ancient materials. Interaction between the development of methods and applications encourage both the emergence of new instruments and an optimized use of spectroscopic techniques and cutting-edge imaging. The building of IPANEMA on the Paris-Saclay site is part of a more global strategy within E-RIHS, the European Research Infrastructure for Heritage Science.

Keywords. Ancient materials, synchrotron, photonic methods, imaging, microsample.

\section{Introduction}

Le synchrotron SOLEIL a ouvert ses premiers instruments aux utilisateurs en janvier 2008. En 2013, les nouveaux locaux d'IPANEMA, l'Institut photonique d'analyse non destructive européen des matériaux anciens, étaient inaugurés par la ministre de la Recherche sur le site du synchrotron. Depuis leur démarrage, SOLEIL et IPANEMA se sont fortement impliqués dans des programmes européens tels que CHARISMA et IPERION CH comme dans des projets bilatéraux.

\section{Qu'est-ce qu'un synchrotron?}

Un synchrotron est une source de lumière intense utilisée pour caractériser la matière sous ses différentes formes à partir de l'interaction photon/matière (fig. 1). C'est à la fois

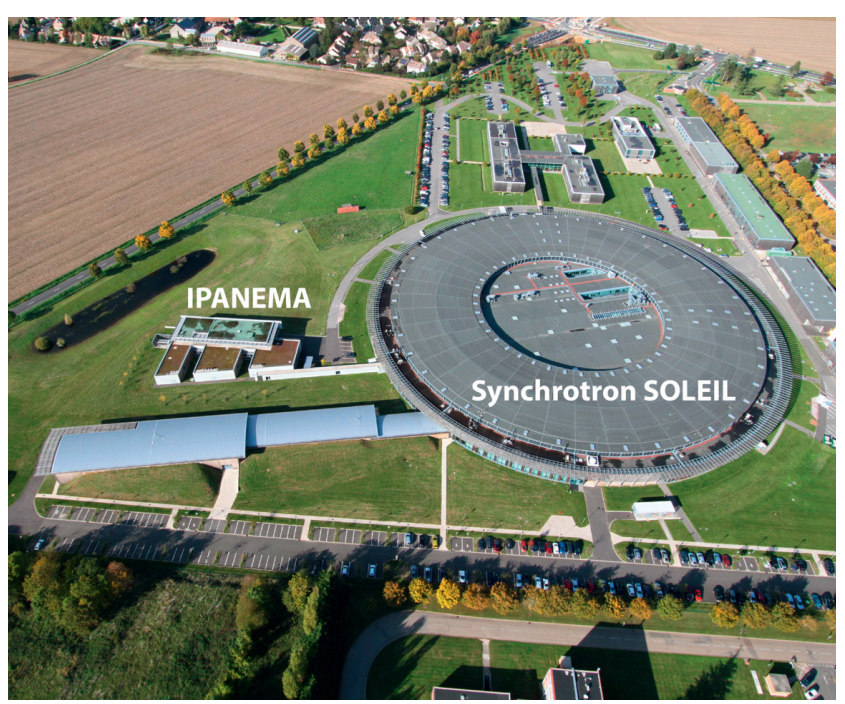

Fig. 1. Le synchrotron SOLEIL et la plateforme IPANEMA attenante. (c) Synchrotron SOLEIL - Cavok Production/L. Persin.

Loïc Bertrand, IPANEMA, CNRS, MCC, UVSQ, Université Paris-Saclay, Synchrotron SOLEIL (loic.bertrand@synchrotron-soleil.fr). Sebastian Schoeder, Synchrotron SOLEIL. Sophie David, IPANEMA, CNRS, MCC, UVSQ, Université Paris-Saclay. Mathieu Thoury, IPANEMA, CNRS, MCC, UVSQ, Université Paris-Saclay. 
une source qui alimente plusieurs dizaines de lignes de caractérisation, ouvrant à plus de cinquante méthodes instrumentales, et un très grand instrument de recherche avec ses instrumental constant, interdisciplinarité et... très forte demande. Depuis une quinzaine d'années ${ }^{1}$, le rayonnement synchrotron est couramment employé pour étudier les matériaux culturels et ceux des collections d'histoire naturelle. Ses propriétés caractéristiques sont sa très grande brillance et son spectre «blanc » couvrant des rayons X à l'infrarouge lointain. Le synchrotron vient donc en complément d'instruments de laboratoire pour l'étude des matériaux anciens, notamment pour l'imagerie 3D, l'imagerie 2D par balayage et la spectroscopie avancée. spécificités : sélection par appel à projets, développement

géométrie du site. Par exemple, le bleu de Prusse est un pigment qui a été très largement employé depuis sa synthèse industrielle au début du XVIII ${ }^{\mathrm{e}}$ siècle. Ce ferrocyanure ferrique comprend des groupements chimiques Fe(II)-CN-Fe(III). La réduction de l'atome de $\mathrm{Fe}$ (III) en $\mathrm{Fe}$ (II) se traduit par une variation de l'énergie d'absorption des photons par le fer, directement observable par cette méthode ${ }^{2}$. D'autres travaux ont porté sur de nouveaux traitements pour le patrimoine bâti ${ }^{3}$ ou les bois archéologiques gorgés d'eau.

IPANEMA collabore depuis 2012 à des projets de recherche dans le cadre du programme Science4arts (financé par l'Agence néerlandaise pour la recherche scientifique). Le projet Paint Alteration in Time (PAinT), par exemple, porté par l'université d'Amsterdam, est dédié à la caractérisation des processus chimiques à micro-échelle qui gouvernent l'altération des peintures à l'huile ; les résultats obtenus devraient permettre de mieux définir des conditions de conservation à même d'enrayer la détérioration.

\section{Archéométrie et technologies anciennes} riaux patrimoniaux. Elle s'est inspirée d'autres initiatives interdisciplinaires en biologie structurale, nanomatériaux, énergies nouvelles. C'est un laboratoire de développement de méthodes avancées de caractérisation de matériaux de l'archéologie, des paléo-environnements, de la paléontologie et du patrimoine culturel, et d'accompagnement de recherches synchrotron, avec des utilisateurs externes hébergés sur la plateforme. Pour ce faire, IPANEMA développe et met à disposition un ensemble de techniques pour préparer les prélèvements, caractériser artefacts et échantillons, analyser statistiquement les jeux de données collectés. Elle apporte un soutien dès le montage d'un projet d'expérience, en complément de l'apport des scientifiques des lignes synchrotron. Les allers-retours entre l'expérience et le développement méthodologique encouragent une fertilisation croisée.

Les matériaux du patrimoine sont particulièrement hétérogènes et présentent souvent des mélanges de composés difficiles à identifier en laboratoire. Pour les matériaux étudiés (avec le soutien de CHARISMA : peinture, bois, matériaux lithiques, papiers), le rayonnement facilite la discrimination des formes chimiques sous lesquelles se présente un composé dans des matériaux du patrimoine. Le plus souvent, l'analyse est réalisée sur une coupe transverse d'un micro-échantillon, ce qui permet d'en étudier la stratigraphie. Sur la période 2009-2014, des équipes européennes ont bénéficié du soutien du programme européen CHARISMA pour des travaux réalisés sur sept lignes différentes du synchrotron.

\section{Conservation et altération}

Les méthodes synchrotron sont particulièrement adaptées à l'étude des mécanismes de dégradation et à l'identification de produits d'altération. En effet, les spectroscopies d'absorption de rayons $\mathrm{X}$ permettent de déterminer la spéciation d'un ion : degré d'oxydation, nature et nombre des atomes voisins,
L'analyse par spectroscopie ou imagerie synchrotron à des échelles micrométriques, voire en deçà, apporte également des informations archéométriques à partir de la détermination de la composition, de la structure ou des propriétés des matériaux. Les modes de circulation d'artefacts peuvent être identifiés à partir de l'analyse d'éléments-traces.

Des travaux ont ainsi porté sur des techniques de coloration de l'or, employées dans la décoration de la châsse médiévale de la collégiale de Notre-Dame de Huy (voir, dans ce volume, l'article de A. Crabbé et al.), ou bien sur la production de décors de cuirs «dorés » (voir, dans ce volume, l'article de Lauriane Robinet et al. $)^{5}$.

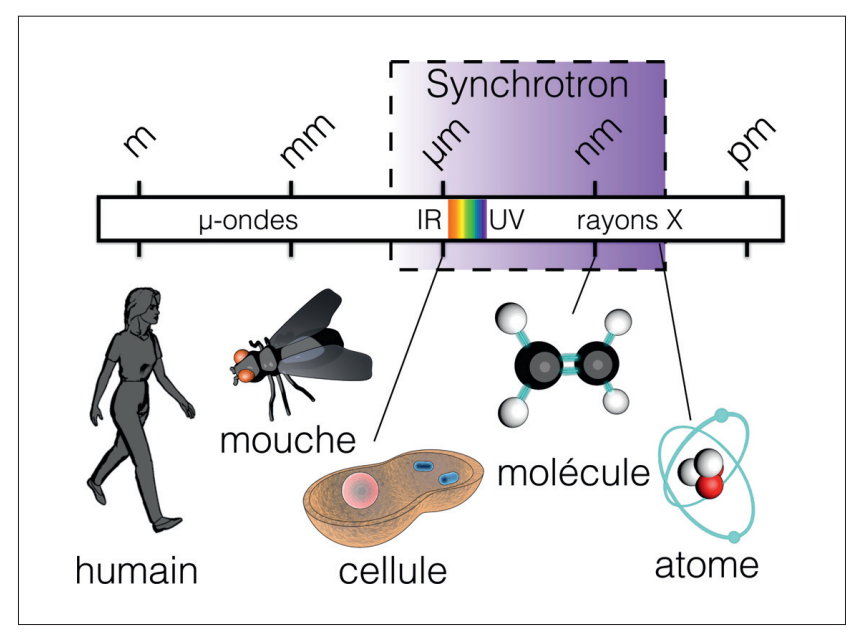

Fig. 2. Gamme d'énergie accessible sur un synchrotron. 
Fig. 3. Le développement de méthodes d'imagerie UV/visible synchrotron permet d'exalter le contraste de composés organiques anciens en microscopie. (C) J.-P. Échard et al. ${ }^{6}$.
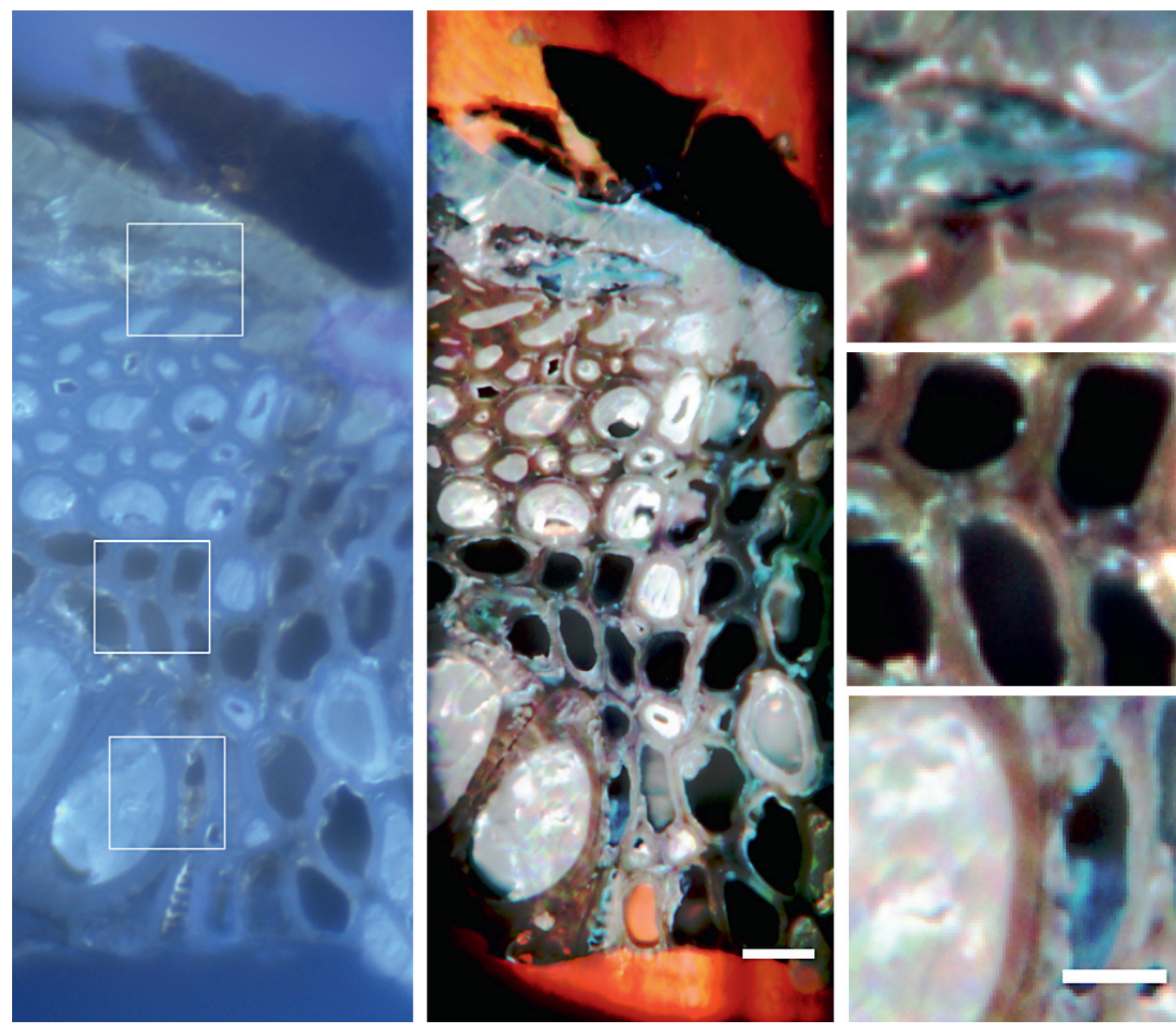

\section{Nouvelles méthodes d'analyse}

Le développement de nouvelles approches d'analyse, notamment d'imagerie spectrale et 3D (fig. 2), est un élément-clé de la démarche d'IPANEMA. Dans le cadre du projet européen CHARISMA, IPANEMA, avec la National Gallery de Londres, a organisé un atelier au format novateur, dédié à la préparation d'échantillons du patrimoine culturel. Il a réuni, sur le site de SOLEIL, une dizaine d'experts venant de plusieurs institutions muséales européennes, qui ont inter-comparé leurs modes de préparation, et établi des recommandations. Cet atelier a été suivi d'une session consacrée à la mise en application de nouvelles procédures de préparation au laboratoire, et à leur évaluation sur la ligne de lumière SMIS.

Dans le cadre d'IPERION CH, IPANEMA est impliquée dans deux thématiques cruciales pour l'analyse synchrotron : le positionnement pour l'analyse de micro-échantillons et la maîtrise des dégâts d'irradiation. Cette dernière était par ailleurs au centre des préoccupations de l'atelier international Investigating Heritage Materials with Safer Ion and Photon Beam Experiments, organisé en décembre 2015 par IPANEMA et le C2RMF sous l'égide de l'AIEA.

\section{Perspectives}

La mise en place de l'infrastructure E-RIHS (European Research Infrastructure for Heritage Science) ouvre de nouvelles perspectives. Un objectif important sera de soutenir les recherches

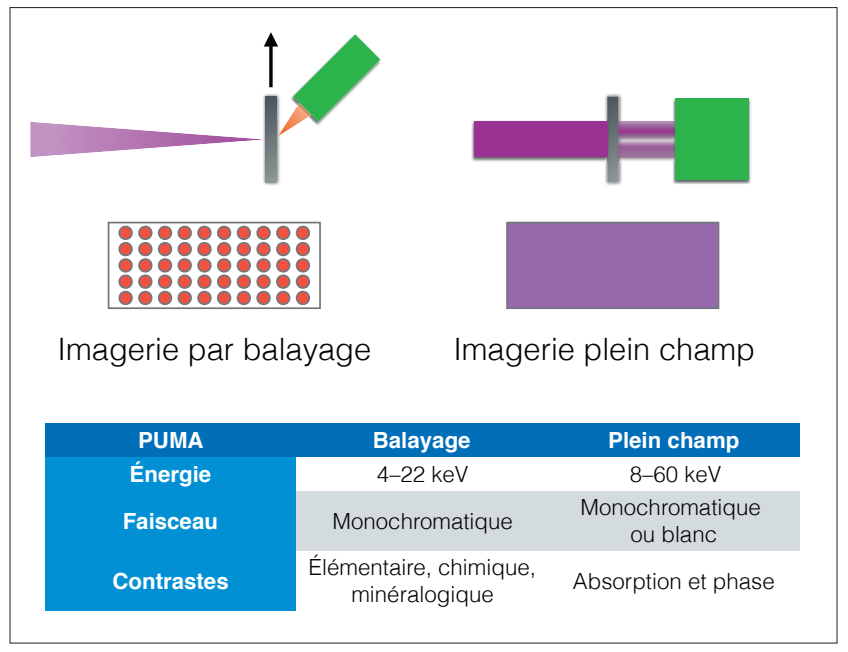

Fig. 4. Modes d'imagerie de la future ligne PUMA optimisée pour les matériaux anciens. 
portant sur de nouvelles méthodologies d'imagerie spectrale et 3D adaptées aux matériaux anciens. À SOLEIL, l'ouverture de la ligne PUMA (Photons Utilisés pour les Matériaux Anciens) sera une étape essentielle pour le développement des sciences du patrimoine.

Notes

1. Bertrand et al., 2012, 2012a.

2. Gervais et al., 2013, 2014.

3. Mudronja et al., 2013, Vanmeert et al., 2013.

4. Crabbé et al., 2013.

5. Bonnot-Diconne et al., 2014.

6. Échard et al., 2015.

\section{Bibliographie}

Bertrand L., Cotte M., Stampanoni M.,

Thoury M., Marone F., Schöder S, 2012,

"Development and trends in

synchrotron studies of ancient and historical materials", Physics Reports, $n^{\circ} 519 / 2$, p. 51-96.

Bertrand L., Robinet L., Thoury M., Janssens K., Cohen S. X., Schöder S., 2012a, "Cultural heritage and archaeology materials studied by synchrotron spectroscopy and imaging", Applied Physics A, n ${ }^{\circ}$ 106/2, p. 377-396.

Bertrand L., Bernard S., Marone F., Thoury M., Reiche I., Gourrier A., Sciau P., Bergmann U., 2016, "Emerging approaches in synchrotron studies of materials from cultural and natural history collections", Topics in Current Chemistry, 374, sous presse.

Bonnot-Diconne C., Robinet L., Pacheco C., Ioele M., Paris M., 2014, "MultiTechnique Analysis of Gilt Leather Wall-Coverings (16th-18th Century)", Proceedings of the ICOM-CC Triennial Meeting, Melbourne, 15-19 septembre 2014, ed. J. Bridgland, Paris, International Council of Museums, art. 0701.

Crabbé A., Languille M.-A., Vandendael I., Hammons J., Silly M., Dewanckel G., Terryn H. Wouters H., 2013,

"Colorando auro: contribution to the understanding of a medieval recipe to colour gilded silver plates", Applied Physics A, n ${ }^{\circ} 111 / 1$, p. 39-46.

Echard J.-P., Thoury M., Berrie B. H., Séverin-Fabiani T., Vichi A., Didier M., Réfrégiers M., Bertrand L., 2015, "Synchrotron DUV luminescence micro-imaging to identify and map historical organic coatings on wood", Analyst, ${ }^{\circ} 140 / 15$, p. 5344-5353.

Gervais C., Languille M.-A., Reguer S., Garnier C., Gillet M., 2014, "Light and anoxia fading of prussian blue dyed textiles", Heritage Science, n ${ }^{\circ}$ 2/1, p. 26.
Gervais C., Languille M.-A., Reguer S., Gillet M., Pelletier S., Garnier C., Vicenzi E. P., Bertrand L., 2013, "Why Does Prussian Blue Fade? Understanding the Role(s) of the Substrate", Journal of Analytical Atomic Spectrometry, ${ }^{\circ}$ 28/10, p. 1600-1609.

Gervais C., Languille M.-A., Reguer S., Gille M., Vicenzi E. P., Chagnot S., Baudelet F., Bertrand L., 2013, “'Live' Prussian blue fading by time-resolved X-ray absorption spectroscopy", Applied Physics A, n ${ }^{\circ} 111 / 1$, p. 15-22.

Mudronja D., Vanmeert F., Hellemans K., Fazinic S., Janssens K., Tibljas D. Rogosic M., Jakovljevic S., 2013, "Efficiency of applying ammonium oxalate for protection of monumental limestone by poultice, immersion and brushing methods", Applied Physics A, $\mathrm{n}^{\circ} 111 / 1$, p. 109-119.

Vanmeert F., Mudronja D., Fazinic S., Janssens K., Tibljas D., 2013, "Semiquantitative analysis of the formation of a calcium oxalate protective layer for monumental limestone using combined micro-XRF and micro-XRPD”, X-Ray Spectrometry, $\mathrm{n}^{\circ} 42 / 4$, p. 256-261. 